\title{
Antioxidants for the Treatment of Retinal Disease: Summary of Recent Evidence
}

\author{
Patrick Wang' \\ Eric K Chin (D) ${ }^{2}$ \\ David Almeida ${ }^{3}$ \\ 'School of Medicine, Queen's University, \\ Kingston, Ontario, Canada; ${ }^{2}$ Retina \\ Consultants of Southern California, \\ Redlands, CA, USA; ${ }^{3}$ Erie Retinal Surgery, \\ Erie, PA, USA
}

\begin{abstract}
Retinal tissue is prone to oxidant burden and oxidative stress secondary to the generation of reactive oxygen species from high metabolic demand. The formation of reactive oxygen species occurs primarily from the mitochondrial respiratory chain as well as several enzymatic and oxidation reactions that occur in the neurosensory retina and retinal pigment epithelium. This oxidative stress has been implicated in the pathogenesis of several retinal diseases and the role of antioxidants as a therapeutic treatment shows promise in slowing the progression of certain diseases. The aim of this narrative review is to describe the mechanisms of retinal oxidative stress and summarize the current available evidence for antioxidants as a treatment for vitreoretinal disorders.
\end{abstract}

Keywords: antioxidant, reactive oxygen species, retina

\section{Introduction}

Reactive oxygen species (ROS) is a collective term encompassing both oxygen free radicals such as superoxide $\left(\mathrm{O}_{2}{ }^{-}\right)$, hydroxyl $(\mathrm{OH})$, peroxyl $\left(\mathrm{RO}_{2}{ }^{-}\right)$, hydroperoxyl $\left(\mathrm{HO}_{2}{ }^{\prime}\right)$, and nonradical oxidizing agents that can be converted into oxygen free radicals such as hydrogen peroxide $\left(\mathrm{H}_{2} \mathrm{O}_{2}\right)$, hypochlorous acid $(\mathrm{HOCl})$, and ozone $\left(\mathrm{O}_{3}\right) .{ }^{1}$ In the retina, ROS arise in a myriad of ways including as a product of oxidative phosphorylation in the mitochondria, in photochemical and enzymatic reactions, and exposure to ultraviolet (UV) light. ${ }^{2,3}$ During normal metabolism, ROS are produced at nascent moderate levels secondary to the cellular metabolism required in maintaining physiological functions such as proliferation, host defense, signal transduction, and gene expression. ${ }^{4}$ However, excessive ROS production resulting from mitochondrial dysfunction and impaired antioxidant defense systems can contribute to several pathophysiological processes in the retina including cellular injury, ischemia, aging, and apoptosis..$^{5-7}$ In these states, the oxidative imbalance between formation and clearance of ROS has been implicated in disease progression and impairing survival signaling.

Under normal physiological conditions, there is a cellular balance between ROS generation and clearance as eukaryotic cells have several antioxidative defense mechanisms. However, when ROS cellular overproduction overwhelms intrinsic antioxidant capacity, a state of oxidative stress results in damage to biomolecules such as DNA. ${ }^{8,9}$ The cell's inability to repair the incurred damage due to decreased antioxidant defense may cause genetically programmed cell death (apoptosis) or mutations in the DNA, which can subsequently lead to carcinogenesis or neurodegeneration. ${ }^{8}$
Correspondence: David Almeida Erie Retinal Surgery, 300 State Street, Suite 20I, Erie, PA, 16507, USA

$\mathrm{Tel}+\mid$ 8I4 456-424I

Fax +I 814 453-3354

Email drpa@pm.me 
Table I Classes of Enzymatic and Nonenzymatic Antioxidants

\begin{tabular}{|l|l|}
\hline $\begin{array}{l}\text { Intracellular Enzymatic } \\
\text { Antioxidants }\end{array}$ & $\begin{array}{l}\text { Nonenzymatic } \\
\text { Antioxidants }\end{array}$ \\
\hline Superoxide dismutase (SODI, SOD2) & Vitamins A, C, and E \\
Catalase & Flavonoids \\
Glutathione peroxidase (GP) & Carotenoids \\
Glutathione reductase (GR) & Glutathione \\
Peroxiredoxin & Plant polyphenols \\
& Uric acid \\
& Theaflavin \\
& Allyl sulfides \\
& Curcumin \\
& Melatonin \\
& Bilirubin \\
& Polyamines \\
\hline
\end{tabular}

Antioxidants may be classified based on activity as enzymatic and nonenzymatic antioxidants. The five major types of intracellular enzymatic antioxidants include copper-zinc superoxide dismutase (Cu/Zn-SOD, SOD1) and manganese superoxide dismutase (Mn-SOD, SOD2), catalase, peroxiredoxin, glutathione peroxidase (GPx), and glutathione reductase (GR). ${ }^{10}$ Both SOD1 and SOD2 convert superoxide to oxygen and hydrogen peroxide, while catalase and GPx convert hydrogen peroxide into water and oxygen. Apart from the antioxidant enzymes, small molecular weight and nonenzymatic antioxidants are also involved in the protection of the intracellular components against ROS. Examples of these include natural nonenzymatic antioxidants such as vitamin E, A, C, flavonoids, carotenoids, glutathione, plant polyphenols, theaflavin, allyl sulfides, curcumin, melatonin, bilirubin, and polyamines (Table 1). ${ }^{11,12}$

Owing to the critical pathogenic determinants of oxidative stress in retinal tissue, therapeutic candidates with antioxidant mechanisms have been central to lead candidate designs and development. However, the evidence for antioxidants in preventing retinal diseases has evolved significantly and here we present a narrative review of the evidence. The objective of this review is to highlight the current landscape of antioxidants as a treatment modality for retinal disease.

\section{Methods}

Our narrative review utilizes literature that describe the role of antioxidants in retinal diseases and were identified by searching the MEDLINE database using a structured search comprising of the following medical subject heading (MeSH) terms and keywords: antioxidant, retina, and therapeutics. Preclinical studies, case reports, case series, observational studies, and randomized controlled trials were considered for inclusion. Searches were undertaken in December 2020 and were time constrained from 2015-2020. Articles regarding established antioxidant therapies published prior to 2015 were identified from a manual search. Articles were also identified from a manual search of reference lists within included articles.

The abstracts of identified articles were screened and classified dichotomously for inclusion or exclusion in the review. To be included in our narrative review, the article must have described the effect of an antioxidant in a retinal disease process or model, published in a peerreviewed journal, written in English and available in full text. One reviewer (PW) read the abstracts independently and articles requiring further clarification were included or excluded through consensus discussion with another reviewer (DA).

The full text of articles that met inclusion criteria were read, then extracted to provide a structured framework for analysis. For each of the included studies we extracted year of publication, disease process, antioxidant agent, mechanism of action, and efficacy.

\section{Results}

Our search and screening strategy resulted in 45 studies describing the role of 15 antioxidants being included in this narrative review.

\section{Antioxidants for Age-related Macular Degeneration (AMD)}

Age-related macular degeneration (AMD) is a complex chronic neurodegenerative and progressive disease characterized by retinal drusenoid deposits, lipofuscin deposition, loss of retinal pigment epithelium (RPE) cells, and choroidal neovascularization ( $\mathrm{CNV}$; neovascular exudative form).$^{46}$ Advanced age and genetic predisposition are the strongest risk factors. ${ }^{46}$ Excessive ROS production is known to play a pivotal role in AMD pathogenesis owing to the evidence of RPE cells as critical targets of oxidative stress. $^{2}$ In AMD, as ROS levels increase, attenuated or compromised antioxidant defense systems produce resultant oxidative stress with photoreceptor, RPE, and choriocapillaris cell death. ${ }^{46,47}$

The aim of the age-related eye disease studies (AREDS, AREDS2) was to investigate the benefit of 
antioxidants native to healthy retinal and macular tissue and assess efficacy of supplementation on progression of dry AMD to more advanced forms. The AREDS randomized trial consisted of 4757 patients who took either AREDS supplements (vitamin C, vitamin E, betacarotene, zinc, copper) or placebo. ${ }^{48}$ The AREDS supplementation group was correlated with strong prevention in disease progression in comparison to the placebo group (OR: 0.68 , 95\%CI: $0.53-0.87$ ). ${ }^{48}$

The aim of the AREDS2 randomized trial was to improve the efficacy and safety profile of the AREDS supplement formulation; specifically, the original formulation of beta-carotene was replaced with lutein and zeaxanthin. ${ }^{49}$ AREDS2 demonstrated a $10 \%$ reduction in the progression of intermediate dry AMD to advanced forms of atrophic and neovascular AMD compared to placebo. ${ }^{49}$ Based on the AREDS and AREDS2 trials, antioxidant supplementation is regarded as a bona fide strategy to mitigate AMD progression; however, the findings of the AREDS trials were based on a relatively wellnourished American population and may not be generalizable to other populations. ${ }^{50}$

Omega-3 fatty acids have exhibited the ability to renew RPE cells and, when deficient, can lead to photoreceptor degradation and accumulation of drusen in both the RPE and sub-RPE space. ${ }^{51}$ There have been several observational studies showing a positive effect of omega-3 fatty acid supplementation as a preventative measure of AMD progression but currently no randomized trials have confirmed these findings. ${ }^{52-54}$

Resveratrol is a phenolic phytochemical derived mainly from plant sources such as Polygonum cuspidatum and Vitis vinifera. ${ }^{29}$ Resveratrol has been demonstrated to suppress UV-induced hydrogen peroxide production in RPE cells. Increase in RPE cell viability was postulated from resveratrol's ability to attenuate ROS production from altered oxidative phosphorylation outside of the mitochondria and specific to the outer segments of rod photoreceptors as well as inhibition of the mitogenactivated protein kinase (MAPK)/ERK1/2 cascade. ${ }^{55-57}$ Similarly, pramipexole, a dopamine receptor agonist, may also protect against light-induced retinal oxidative damage by increasing ROS scavenging activity and decreasing caspase activity. ${ }^{58}$

Edaravone is a free radical scavenger and a drug used to treat acute ischemic stroke. ${ }^{23}$ Edaravone has shown to be effective against retinal degeneration both in vivo and in vitro models. ${ }^{59-61}$ The mechanism mediated by edaravone occurs via the reduction of ROS, lipid peroxidation, and VEGF-induced endothelial cell proliferation. In an UV light-induced neovascular AMD mice model, edaravone administered intravenously reduced $\mathrm{CNV}$ area and vascular leakage. ${ }^{60}$

\section{Antioxidants for Diabetic Retinopathy}

Diabetic retinopathy (DR) is a chronic progressive complication of diabetes mellitus type 1 or type 2 characterized by retinal neurodegeneration in the setting of chronic diabetes. ${ }^{25}$ Diabetic retinopathy, a microvascular diabetes complication resulting from capillary damage, demonstrates pericyte and endothelial cell loss by accelerated apoptosis; consequently, this reduction of pericyte numbers produces hallmarks of degeneration including: presence of ghost cells, increased numbers of acellularoccluded capillaries, development of microaneurysms, and capillary basement membrane thickening. ${ }^{26,27}$

Hyperglycemia, the main driving force of diabetic retinal disease and DR, leads to an array of metabolic and functional derangements in retinal vascular and neuronal cells including overproduction of mitochondrial ROS. ${ }^{26,28}$ The chronic increase in local oxidative stress disrupts retinal metabolism and accelerates premature endothelial cell apoptosis via mitochondrial dysfunction typical in $\mathrm{DR}^{28}$ Ultimately, endothelial cell loss results in a compromised blood-retinal barrier resulting in exudation, macular edema and ischemia.

Resveratrol, in addition to the possible therapeutic potential is AMD described above, has been implicated in the inhibition of many pro-oxidant pathways involved in the pathogenesis of diabetic retinopathy through in vitro models involving PI3K/AKT, AMPK, Sirt1, PGC-1 $\alpha$, miR-29b, TGF- $\beta$, PKC $\beta$, COX-2, MEK/ERK, interleukin 6 (IL6), interleukin 8 (IL8) and vascular endothelial growth factor (VEGF) markers. ${ }^{30-33}$ There has also been several in vivo models looking at the effects of resveratrol in STZ-induced mice which demonstrate reduced STZinduced retinal cell apoptosis and superoxide dismutase activity. $^{34,35}$

Metanx ${ }^{\circledR}$, a vitamin $B$ supplement consisting of a combination of the active components of vitamins $\mathrm{B}_{6}$, $\mathrm{B}_{9}$, and $\mathrm{B}_{12}$ has been demonstrated to reduce diabetesinduced retinal superoxide generation in mouce models. ${ }^{36}$ However, in this model Metanx ${ }^{\circledR}$ was unable to inhibit degeneration of retinal capillaries or capillary pericytes which suggest that oxidative stress may not be directly implicated in the pathogenesis of these lesions. ${ }^{36}$ Further 
evidence demonstrates a lower incidence of diabetic retinopathy in the Japanese type 2 diabetes population. ${ }^{37}$ This may be attributed to the activity of vitamin $\mathrm{B}_{6}$ to scavenge superoxide radicals and prevent lipid peroxidation processes that generate ROS. Retinol, also known as vitamin $\mathrm{A}_{1}$, was associated with a $17 \%$ lower risk of diabetic retinopathy in a Japanese population with a $100 \mu \mathrm{g}$ /day higher dietary retinol intake. ${ }^{38}$

There is a growing interest in exploration of novel therapeutic targets for the management of DR such as the role of miRNA; specifically, miR-145 has been explored as a novel regulator in retinal endothelial cells subject to high glucose environments. In this model, overexpression of miR-145 serves a protective role for retinal endothelial cells from apoptosis and oxidative stress, by targeting TLR4 signaling. ${ }^{39}$ Moreover, miR-126 expression has also been implicated in induced vascular restoration through Niaspan ${ }^{\circledR}$ (naicin) treatment in diabetic retinopathy rat models. ${ }^{40}$

Crocin, the active anti-inflammatory antioxidant in saffron has been attributed to neuroprotective effects and increases retinal blood flow. One RCT compared the therapeutic effects of crocin on refractory DME in 68 patients. ${ }^{41}$ Reported visual acuity and macular thickness improved in the crocin treatment groups, attributed to reduction of inflammatory damage caused by oxidative stress. This may be through activation of the PI3K/Akt signaling pathway which is known to provide significant protection of neural cells against premature cell death and apoptosis. $^{42}$

Ubiquinone, also known as coenzyme Q10, is one of the first lines of defense against oxidative damage of the mitochondria and oxidation of low-density lipoproteins. ${ }^{43}$ In one randomized trial with 60 patients, combination therapy with the addition of antioxidants $(10 \mathrm{mg}$ lutein, $4 \mathrm{mg}$ astaxanthin, $1 \mathrm{mg}$ zeaxanthin, $180 \mathrm{mg}$ vitamin C, $30 \mathrm{mg}$ vitamin E, $20 \mathrm{mg}$ zinc, $1 \mathrm{mg}$ copper) showed improvements in mitochondrial homeostasis and diminished energy catabolism produced through oxidative damage in collected blood samples. ${ }^{43}$

The effects of proanthocyanidin extract, one of the main active components of grape seed oil, showed a significantly greater improvement in DR severity compared to placebo. ${ }^{44}$ However, no significant differences existed between groups in optical coherence tomography parameters macular thickness and total macular volume. ${ }^{44}$ Naturally occurring carotenoids such as lutein have not demonstrated any positive effect in visual acuity nonproliferative DR as shown in a retrospective study providing lutein supplementation for four months. ${ }^{45}$

\section{Antioxidants for Other Retinal Diseases Proliferative Vitreoretinopathy (PVR)}

Recent findings indicate that saffron carotenoid constituents, crocins and crocetin, significantly inhibit proliferative vitreoretinopathy development in rabbit models. ${ }^{62}$ Crocetin demonstrates a neuroprotective effect by counteracting retinal oxidative damage, inflammation and protecting retinal cells from subsequent apoptosis. There were no signs of retinal toxicity in these early disease models.

\section{Retinitis Pigmentosa (RP)}

Vitamin A supplementation may be considered to potentially slow loss of retinal function in children. ${ }^{63}$ A prospective observational study compared two cohorts, a cohort receiving vitamin A and a control cohort. The vitamin A cohort experienced a statistically significant reduction in rate of cone ERG amplitude decline during follow-up than the control cohort which exceeded that from the adult clinical trial. ${ }^{63,64}$

\section{Central Serous Chorioretinopathy (CSCR)}

A multicenter randomized controlled study investigating the functional and morphological changes in 100 patients with central serous chorioretinopathy with supplementation of lutein has shown patients who received lutein supplementation had significant reduction in mean subfoveal fluid thickness and improvement in vision outcomes. ${ }^{65}$

\section{Ophthalmic Antioxidants Unrelated to Retinal Disease: Glaucoma}

Glaucoma is an optic neuropathy characterized by progressive degeneration of retinal ganglion cells (RGCs). Many modern treatment approaches have focused on reduction of increased intraocular pressure (IOP). Elevated IOP in the pathogenesis of glaucoma has been shown to increase endogenous ROS within the trabecular meshwork and abnormal mitochondria function in RGCs through various intracellular pathways. ${ }^{13,14}$ This increase of ROS production has been postulated to create an imbalance between pro-oxidative and antioxidant capacity and may serve as crucial factors in early cell injury. ${ }^{15,16}$

Oxidative stress can inflict damage by acting as a second messenger or modulating the protein function by redox modifications and may serve as an early signal triggering neuron injury. ${ }^{14,16,17}$ Oxidative stress-induced 
signaling for neuroinflammation in glaucoma includes the stimulation of a transcriptional program for inflammatory mediators such as nuclear factor-kappa B (NF- $\kappa \mathrm{B}) .{ }^{10} \mathrm{As}$ such, targeting treatment to provide immunomodulation and degenerative neuroprotection may be promising.

Preclinical trials of neuroactive steroid hormones such as progesterone and estradiol have been implicated in the treatment of chronic neurodegenerative diseases due to their protective effects on the mitochondria during times of stress. Specifically, 17 $\beta$-estradiol has been demonstrated to inhibit ROS production, preserve adenosine triphosphate (ATP) production, and decrease mitochondrial calcium loading. ${ }^{18}$ These mechanisms lead to significant neuroprotection in RGCs shown with in vivo models of glaucoma. ${ }^{18,19}$ Thus, estrogen may be a potential target for therapy for preventing ROS-associated neurodegeneration characteristic of glaucoma. Moreover, progesterone has also been demonstrated to decrease concentrations of malondialdehyde (a biochemical marker for oxidative stress) in retinal degeneration 1 (rd1) mice. ${ }^{20}$

Similarly, Eucommia ulmoides extract is a plant containing low molecular weight polyphenols known as lignans and has been shown to have a neuroprotective effect in RGCs exposed to hydrogen peroxide in rat models. ${ }^{21}$ This is accomplished through upregulation of ROSscavenging activity of enzymes including superoxide dismutase (SOD), glutathione peroxidase, and catalase. ${ }^{21}$

Two endogenous antioxidants, trolox and deferoxamine have demonstrated ROS scavenging activity and associated neuroprotective effects of RGCs with in vitro disease models. $^{22}$ Trolox, a water-soluble analog of vitamin $\mathrm{E}$, has been shown to reduce cell death caused by hydrogen peroxide. It is postulated that this is accomplished through a direct mechanism where hydrogen peroxide is converted by reverse dismutation into superoxide. Deferoxamine, an iron chelator, also acts on hydrogen peroxide by preventing its conversion into hydroxyl anion free radicals via inhibition of the Fenton reaction (catalytic process that converts hydrogen peroxide into hydroxyl radicals). ${ }^{22}$ Trolox and deferoxamine were also found to reduce RGC cell death caused by generation of superoxide anions. ${ }^{22}$

Intravitreous injections of edaravone was demonstrated to significantly protect against the NMDA-induced reduction of retinal thickness as well as decreased RGC death induced by oxygen-glucose deprivation (OGD) stress in an in vitro ischemia-reperfusion injury model. ${ }^{24}$

\section{An Approach to Novel Antioxidant Therapeutics for Retinal and Macular Disease}

Oxidative stress, secondary to the pathologic imbalance between oxygen metabolism and antioxidant defense systems, is common in the macula because of the retina's high consumption of oxygen, high proportion of polyunsaturated fatty acids, and exposure to visible light. Our review details the instrumental role of oxidative stress in the pathogenesis of various retinal macular diseases. Furthermore, it follows that agents with antioxidant mechanisms of action may serve both preventative and therapeutic potential.

Fundamentally, the constant oxidation reduction state of the macula is actively regulated by various antioxidant signaling pathways optimized to function in concert with existing oxidative burden. ${ }^{1,12}$ Notwithstanding, when these antioxidant defense systems are compromised, the oxidative burden directly produces retinal disease that is most damaging to the macula resulting in the pathogenic endpoints of cell death, apoptosis and neurodegeneration.

As elucidated in this review, novel small molecule antioxidant treatments for macular disease like dry and neovascular AMD have demonstrated efficacy within in vitro, in vivo, and preclinical disease models but few have been tested in later phase clinical studies. ${ }^{2,46,49,52,59,61,66}$ This represents a knowledge gap regarding the clinical evidence needed to proceed with the development of new efficacious antioxidant treatments. Still, when we consider the evidence reviewed here, we can state that the most promising treatments will be those that prevent apoptosis induced by oxidative stress. ${ }^{2,46,52}$ It is our hope that novel candidates with specific targets will be investigated to impact critical clinical outcomes.

\section{Funding}

The authors received no financial support for the research, authorship and/or publication of this article.

\section{Disclosure}

EC: Consultant (Alcon, Allergan, Amgen, Bayer, Chengdu Kanghong Biotechnology, Genentech, IVERIC, Kodiak, Novartis, Ophthotech, Opthea); Cofounder/Equity Holder (Citrus Therapeutics). DA: Advisory Board, Consultant, Speaker (Alcon, Alimera, Allergan, Bayer, Genentech, Novartis, Regeneron); Cofounder, Equity Holder (Citrus 
Therapeutics). The authors report no other conflicts of interest in this work.

\section{References}

1. Halliwell B, Gutteridge JMC. Free Radicals in Biology and Medicine. 2015. doi:10.1093/acprof:oso/9780198717478.001.0001

2. Jarrett SG, Boulton ME. Consequences of oxidative stress in age-related macular degeneration. Mol Aspects Med. 2012;33 (4):399-417. doi:10.1016/j.mam.2012.03.009

3. Roehlecke C, Schumann U, Ader M, Knels L, Funk RHW. Influence of blue light on photoreceptors in a live retinal explant system. Mol Vis. 2011;17:876-884.

4. Bardaweel SK, Gul M, Alzweiri M, Ishaqat A, Alsalamat HA, Bashatwah RM. Reactive oxygen species: the dual role in physiological and pathological conditions of the human body. Eurasian J Med. 2018;50(3):193-201. doi:10.5152/eurasianjmed.2018.17397

5. Zhao L, Feng Z, Zou X, Cao K, Xu J, Liu J. Aging leads to elevation of O-GlcNAcylation and disruption of mitochondrial homeostasis in retina. Oxid Med Cell Longev. 2014;2014:1-11. doi:10.1155/2014/ 425705

6. Kaur C. Hypoxia-ischemia and retinal ganglion cell damage. Clini Ophthalmol. 2008;879. doi:10.2147/opth.s3361

7. Gil J, Almeida S, Oliveira CR, Rego AC. Cytosolic and mitochondrial ROS in staurosporine-induced retinal cell apoptosis. Free Radic Biol Med. 2003;35(11):1500-1514. doi:10.1016/j.freeradbiomed.20 03.08.022

8. Evans MD, Dizdaroglu M, Cooke MS. Oxidative DNA damage and disease: induction, repair and significance. Mutat Res Rev Mutat Res. 2004. doi:10.1016/j.mrrev.2003.11.001

9. Valko M, Leibfritz D, Moncol J, Cronin MTD, Mazur M, Telser J. Free radicals and antioxidants in normal physiological functions and human disease. Int $J$ Biochem Cell Biol. 2007;39(1):44-84. doi:10.1016/j.biocel.2006.07.001

10. Dröge W. Free radicals in the physiological control of cell function. Physiol Rev. 2002;82(1):47-95. doi:10.1152/physrev.00018.2001

11. Mirończuk-Chodakowska I, Witkowska AM, Zujko ME. Endogenous non-enzymatic antioxidants in the human body. Adv Med Sci. 2018;63(1):68-78. doi:10.1016/j.advms.2017.05.005

12. Nimse SB, Pal D. Free radicals, natural antioxidants, and their reaction mechanisms. RSC Adv. 2015;5(35):27986-28006. doi:10.1039/ c4ra13315c

13. He Y, Leung KW, Zhang YH, et al. Mitochondrial complex i defect induces ROS release and degeneration in trabecular meshwork cells of POAG patients: protection by antioxidants. Invest Ophthalmol Vis Sci. 2008;49(4):1447. doi:10.1167/iovs.07-1361

14. Tezel G. Oxidative stress in glaucomatous neurodegeneration: mechanisms and consequences. Prog Retin Eye Res. 2006;25 (5):490-513. doi:10.1016/j.preteyeres.2006.07.003

15. Izzotti A, Bagnis A, Saccà SC. The role of oxidative stress in glaucoma. Mutat Res Rev Mutat Res. 2006;612(2):105-114. doi:10.1016/j.mrrev.2005.11.001

16. Nita M, Grzybowski A. The role of the reactive oxygen species and oxidative stress in the pathomechanism of the age-related ocular diseases and other pathologies of the anterior and posterior eye segments in adults. Oxid Med Cell Longev. 2016;2016:1-23. doi:10.1155/2016/3164734

17. Von Thun Und Hohenstein-Blaul N, Kunst S, Pfeiffer N, Grus FH. Basic biochemical processes in glaucoma progression. Der Ophthalmologe. 2015;112(5):395-401. doi:10.1007/s00347-015-00 07-9

18. Hao M, Li Y, Lin W, et al. Estrogen prevents high-glucose-induced damage of retinal ganglion cells via mitochondrial pathway. Graefes Arch Clin Exp Ophthalmol. 2014;253(1):83-90. doi:10.1007/s00417014-2771-7
19. Prokai-Tatrai K, Xin H, Nguyen V, et al. 17ß-Estradiol eye drops protect the retinal ganglion cell layer and preserve visual function in an in vivo model of glaucoma. Mol Pharm. 2013;10(8):3253-3261. doi:10.1021/mp400313u

20. Sánchez-Vallejo V, Benlloch-Navarro S, López-Pedrajas R, Romero FJ, Miranda M. Neuroprotective actions of progesterone in an in vivo model of retinitis pigmentosa. Pharmacol Res. 2015;99:276-288. doi:10.1016/j.phrs.2015.06.019

21. Li CP, Qiu GZ, Liu B, Chen JL, Fu HT. Neuroprotective effect of lignans extracted from Eucommia ulmoides Oliv. on glaucoma-related neurodegeneration. Neurol Sci. 2016;37(5):755-762. doi:10.1007/ s10072-016-2491-3

22. Schlieve CR, Lieven CJ, Levin LA. Biochemical activity of reactive oxygen species scavengers do not predict retinal ganglion cell survival. Invest Ophthalmol Vis Sci. 2006;47(9):3878. doi:10.1167/ iovs.05-1010

23. Lapchak PA. A critical assessment of edaravone acute ischemic stroke efficacy trials: is edaravone an effective neuroprotective therapy? Expert Opin Pharmacother. 2010;11(10):1753-1763. doi:10.1517/14656566.2010.493558

24. Masuda T, Shimazawa M, Hara H. Retinal diseases associated with oxidative stress and the effects of a free radical scavenger (edaravone). Oxid Med Cell Longev. 2017;2017:1-14. doi:10.1155/ 2017/9208489

25. Lee R, Wong TY, Sabanayagam C. Epidemiology of diabetic retinopathy, diabetic macular edema and related vision loss. Eye Vis. 2015;2(1). doi:10.1186/s40662-015-0026-2

26. Kowluru RA, Mishra M. Oxidative stress, mitochondrial damage and diabetic retinopathy. Biochim Biophys Acta. 2015;1852 (11):2474-2483. doi:10.1016/j.bbadis.2015.08.001

27. Behl T, Kaur I, Kotwani A. Implication of oxidative stress in progression of diabetic retinopathy. Surv Ophthalmol. 2016;61 (2):187-196. doi:10.1016/j.survophthal.2015.06.001

28. Giacco F, Brownlee M, Schmidt AM. Oxidative stress and diabetic complications. Circ Res. 2010;107(9):1058-1070. doi:10.1161/ CIRCRESAHA.110.223545

29. Bola C, Bartlett H, Eperjesi F. Resveratrol and the eye: activity and molecular mechanisms. Graefes Arch Clin Exp Ophthalmol. 2014;252(5):699-713. doi:10.1007/s00417-014-2604-8

30. Li J, Yu S, Ying J, Shi T, Wang P. Resveratrol prevents ROSinduced apoptosis in high glucose-treated retinal capillary endothelial cells via the activation of AMPK/Sirt1/PGC-1 $\alpha$ pathway. Oxid Med Cell Longev. 2017;2017:1-10. doi:10.1155/2017/ 7584691

31. Zeng K, Wang Y, Yang N, et al. Resveratrol inhibits diabetic-induced Müller cells apoptosis through microRNA-29b/specificity protein 1 pathway. Mol Neurobiol. 2017;54(6):4000-4014. doi:10.1007/s120 35-016-9972-5

32. Soufi FG, Mohammad-nejad D, Ahmadieh H. Resveratrol improves diabetic retinopathy possibly through oxidative stress - nuclear factor кB - apoptosis pathway. Pharmacol Rep. 2012;64(6):1505-1514. doi:10.1016/S1734-1140(12)70948-9

33. Chan C-M, Chang -H-H, Wang V-C, Huang C-L, Hung C-F, Yang C-M. Inhibitory effects of resveratrol on PDGF-BB-induced retinal pigment epithelial cell migration via PDGFR $\beta$, PI3K/Akt and MAPK pathways. PLoS One. 2013;8(2):e56819. doi:10.1371/journal. pone. 0056819

34. Soufi FG, Vardyani M, Sheervalilou R, Mohammadi M, Somi MH. Long-term treatment with resveratrol attenuates oxidative stress pro-inflammatory mediators and apoptosis in streptozotocin-nicotinamide-induced diabetic rats. Gen Physiol Biophys. 2012;31(04):431-438. doi:10.4149/gpb_2012_039

35. Kim YH, Kim YS, Roh GS, Choi WS, Cho GJ. Resveratrol blocks diabetes-induced early vascular lesions and vascular endothelial growth factor induction in mouse retinas. Acta Ophthalmol. 2012;90(1):e31-e37. doi:10.1111/j.1755-3768.2011.02243.x 
36. Liu H, Tang J, Allen Lee C, Kern TS. Metanx and early stages of diabetic retinopathy. Invest Ophthalmol Vis Sci. 2015. doi:10.1167/ iovs. 14-15220

37. Horikawa C, Yoshimura Y, Kamada C, et al. Dietary sodium intake and incidence of diabetes complications in Japanese patients with type 2 diabetes: analysis of the Japan diabetes complications study (JDCS). $J$ Clin Endocrinol Metab. 2014;99(10):3635-3643. doi:10.1210/jc.2013-4315

38. Zhang C, Li K, Zhang J, et al. Relationship between retinol and risk of diabetic retinopathy: a Case-Control Study. Asia Pac J Clin Nutr. 2019. doi:10.6133/apjen.201909 28(3).0021

39. Hui Y, Yin Y. MicroRNA-145 attenuates high glucose-induced oxidative stress and inflammation in retinal endothelial cells through regulating TLR4/NF-кB signaling. Life Sci. 2018;207:212-218. doi:10.1016/j.1fs.2018.06.005

40. Wang Y, Yan H. MicroRNA-126 contributes to Niaspan treatment induced vascular restoration after diabetic retinopathy. Sci Rep. 2016. doi:10.1038/srep26909

41. Sepahi S, Mohajeri SA, Hosseini SM, et al. Effects of crocin on diabetic maculopathy: a placebo-controlled randomized clinical trial. Am J Ophthalmol. 2018;190:89-98. doi:10.1016/j.ajo.2018.03.007

42. Yang X, Huo F, Liu B, et al. Crocin inhibits oxidative stress and pro-inflammatory response of microglial cells associated with diabetic retinopathy through the activation of PI3K/Akt signaling pathway. J Mol Neurosci. 2017;61(4):581-589. doi:10.1007/s12031017-0899-8

43. Rodríguez-Carrizalez AD, Castellanos-González JA, MartínezRomero EC, et al. The antioxidant effect of ubiquinone and combined therapy on mitochondrial function in blood cells in non-proliferative diabetic retinopathy: a Randomized, Double-Blind, Phase IIA, Placebo-Controlled Study. Redox Rep. 2016. doi:10.1179/ 1351000215Y.0000000032

44. Moon SW, Shin YU, Cho H, Bae SH, Kim HK. Effect of grape seed proanthocyanidin extract on hard exudates in patients with non-proliferative diabetic retinopathy. Medicine. 2019;98(21): e15515. doi:10.1097/MD.0000000000015515

45. Ren YB, Qi YX, Su XJ, Luan HQ, Sun Q. Therapeutic effect of lutein supplement on non-proliferative diabetic retinopathy: a Retrospective Study. Medicine. 2019;98(29):e15404. doi:10.1097/ MD.0000000000015404

46. Hernández-Zimbrón LF, Zamora-Alvarado R, Ochoa-de La Paz L, et al. Age-related macular degeneration: new paradigms for treatment and management of AMD. Oxid Med Cell Longev. 2018;2018:1-14. doi:10.1155/2018/8374647

47. Datta S, Cano M, Ebrahimi K, Wang L, Handa JT. The impact of oxidative stress and inflammation on RPE degeneration in non-neovascular AMD. Prog Retin Eye Res. 2017;60:201-218. doi:10.1016/j.preteyeres.2017.03.002

48. Kassoff A, Kassoff J, Buehler J, et al. A randomized, placebo-controlled, clinical trial of high-dose supplementation with vitamins $\mathrm{C}$ and $\mathrm{E}$, beta carotene, and zinc for age-related macular degeneration and vision loss: AREDS report no. 8. Arch Ophthalmol. 2001. doi:10.1001/archopht.119.10.1417

49. Age-Related Eye Disease Study 2 (AREDS2) Research Group. Lutein + zeaxanthin and omega-3 fatty acids for age-related macular degeneration. JAMA. 2013. doi:10.1001/jama.2013.4997

50. Mukhtar S, Ambati BK. The value of nutritional supplements in treating age-related macular degeneration: a review of the literature. Int Ophthalmol. 2019;39(12):2975-2983. doi:10.1007/s10792-01901140-6

51. Lawrenson JG, Evans JR. Omega 3 fatty acids for preventing or slowing the progression of age-related macular degeneration. Cochrane Database Syst Rev. 2015. doi:10.1002/14651858. CD010015.pub3
52. Chua B, Flood V, Rochtchina E, Wang JJ, Smith W, Mitchell P. Dietary fatty acids and the 5-year incidence of age-related maculopathy. Arch Ophthalmol. 2006;124(7):981. doi:10.1001/ archopht.124.7.981

53. Georgiou T, Neokleous A, Nicolaou D, Sears B. Pilot study for treating dry age-related macular degeneration (AMD) with high-dose omega-3 fatty acids. PharmaNutrition. 2014;2(1):8-11. doi:10.1016/j.phanu.2013.10.001

54. Seddon JM, George S, Rosner B. Cigarette smoking, fish consumption, omega-3 fatty acid intake, and associations with age-related macular degeneration: the US Twin Study of age-related macular degeneration. Arch Ophthalmol. 2006;124(7):995. doi:10.1001/ archopht.124.7.995

55. Kang JH, Choung SY. Protective effects of resveratrol and its analogs on age-related macular degeneration in vitro. Arch Pharm Res. 2016;39(12):1703-1715. doi:10.1007/s12272-016-0839-0

56. Calzia D, Oneto M, Caicci F, et al. Effect of polyphenolic phytochemicals on ectopic oxidative phosphorylation in rod outer segments of bovine retina. Br J Pharmacol. 2015;172(15):3890-3903. doi:10. 1111/bph. 13173

57. King RE, Kent KD, Bomser JA. Resveratrol reduces oxidation and proliferation of human retinal pigment epithelial cells via extracellular signal-regulated kinase inhibition. Chem Biol Interact. 2005;151 (2):143-149. doi:10.1016/j.cbi.2004.11.003

58. Shibagaki K, Okamoto K, Katsuta O, Nakamura M. Beneficial protective effect of pramipexole on light-induced retinal damage in mice. Exp Eye Res. 2015;139:64-72. doi:10.1016/j.exer.2015.07.007

59. Shimazaki H, Hironaka K, Fujisawa T, et al. Edaravone-loaded liposome eyedrops protect against light-induced retinal damage in mice. Invest Ophthalmol Vis Sci. 2011;52(10):7289. doi:10.1167/iovs.11-7983

60. Imai S, Inokuchi Y, Nakamura S, Tsuruma K, Shimazawa M, Hara H. Systemic administration of a free radical scavenger, edaravone, protects against light-induced photoreceptor degeneration in the mouse retina. Eur J Pharmacol. 2010;642(1-3):77-85. doi:10.1016/j.ejphar. 2010.05.057

61. Masuda T, Shimazawa M, Takata S, Nakamura S, Tsuruma K, Hara $\mathrm{H}$. Edaravone is a free radical scavenger that protects against laser-induced choroidal neovascularization in mice and common marmosets. Exp Eye Res. 2016;146:196-205. doi:10.1016/j. exer.2016.03.020

62. Wang HF, Ma JX, Shang QL, An JB, Chen HT, Wang CX. Safety, pharmacokinetics, and prevention effect of intraocular crocetin in proliferative vitreoretinopathy. Biomed Pharmacother. 2019;10 9:1211-1220. doi:10.1016/j.biopha.2018.10.193

63. Berson EL, Weigel-DiFranco C, Rosner B, Gaudio AR, Sandberg MA. Association of vitamin A supplementation with disease course in children with retinitis pigmentosa. JAMA Ophthalmol. 2018;136(5):490. doi:10.1001/jamaophthalmol.2018.0590

64. Berson EL, Rosner B, Sandberg MA, et al. A randomized trial of vitamin A and vitamin E supplementation for retinitis pigmentosa. Arch Ophthalmol. 1993. doi:10.1001/archopht.1993.01090060049 022

65. Shinojima A, Sawa M, Sekiryu T, et al. A multicenter randomized controlled study of antioxidant supplementation with lutein for chronic central serous chorioretinopathy. Ophthalmologica. 2017;237(3):159-166. doi:10.1159/000455807

66. Bhatt P, Fnu G, Bhatia D, Shahid A, Sutariya V. Nanodelivery of resveratrol-loaded PLGA nanoparticles for age-related macular degeneration. AAPS PharmSciTech. 2020;21(8). doi:10.1208/s122 49-020-01836-4 


\section{Publish your work in this journal}

Clinical Ophthalmology is an international, peer-reviewed journal covering all subspecialties within ophthalmology. Key topics include: Optometry; Visual science; Pharmacology and drug therapy in eye diseases; Basic Sciences; Primary and Secondary eye care; Patient Safety and Quality of Care Improvements. This journal is indexed on PubMed
Central and CAS, and is the official journal of The Society of Clinical Ophthalmology (SCO). The manuscript management system is completely online and includes a very quick and fair peer-review system, which is all easy to use. Visit http://www.dovepress.com/ testimonials.php to read real quotes from published authors.

Submit your manuscript here: https://www.dovepress.com/clinical-ophthalmology-journal 